\title{
Helium atom in an external electric field: Exact diagonalization
}

\author{
T.N. Antsygina and K.A. Chishko \\ B. Verkin Institute for Low Temperature Physics and Engineering of the National Academy of Sciences of Ukraine \\ 47 Lenin Ave., Kharkov 61103, Ukraine \\ E-mail: chishko@ilt.kharkov.ua
}

Received April 9, 2014, published online July 21, 2014

\begin{abstract}
An exact diagonalization method is applied to solve the quantum-mechanical problem of spinless helium atom in an external electric field of arbitrary magnitude. The basis set for two-electron problem is built from different pair combinations $\psi_{n_{a} l_{a} m_{a}}\left(\alpha \mathbf{r}_{a}\right) \psi_{n_{b} l_{b} m_{b}}\left(\alpha \mathbf{r}_{b}\right)$ of orthonormalized single-particle hydrogen-like wave functions $\psi_{n m l}(\mathbf{r})$ belonging to any possibly bound states of the individual $a$ - and $b$-electrons in the Coulomb central field renormalized by the scale parameter $\alpha>0$. Within the selected basis the matrix elements of the total Hamiltonian allows an exact analytical representation in the form of finite numerical sums. The diagonalization procedure is performed by Jacobi algorithm for $N \times N$ square Hermitian matrix built on the basis of dimension $N=25$. The systematics and the numerical values of the low-lying energy levels at zero field are in good agreement with known experimental data. The field dependences of low-lying levels (Stark effect) and polarizability in the ground state of helium atom are presented. It is shown that even extremely high external fields lead only to shifting or splitting of existing low levels, without disturbance of their systematics. Typically, no new lowenergy excitation can be created under external electric field of moderate intensity. Radical reconstruction in spectrum of individual helium atoms can be expected in condensed helium phases where each atom is deeply affected by interaction fields from neighbors. This result should be taken into account at interpretation of electrodynamic experiments on superfluid helium.
\end{abstract}

PACS: 31.15.ac High-precision calculations for few-electron (or few-body) atomic systems.

Keywords: helium atom, two-electron shell, exact diagonalization.

\section{Introduction}

Unusual response of superfluid helium under external electromagnetic exposure is a subject of intensive discussions over last decade [1-8]. The most impressive thing is detection of a certain low-frequency mode of $\sim 180.3 \mathrm{GHz}$ which behaves like specific eigenstate of superfluid flow in ${ }^{4} \mathrm{He}[7,8]$. This mode can be splitted by an external stationary and uniform electric field, and such a splitting increases linearly with applied field $[7,8]$. The nature of the low-frequency mode is still not quite understood, but it is evident that the corresponding phenomena can not be interpreted properly without correct solution of the quantum mechanical problem on helium atom in an external electric field of arbitrary intensity. Standard approach for description of Stark effect in helium based on perturbation theory [9] does not allow us to accomplish the necessary treatment because of restrictions on the magnitude of the external field connected with typical conditions of the perturbation smallness. The corresponding solution can be built within exact diagonalization method, and we realize this program in the present paper.

Two-electron helium atom is the simplest three-body atomic system absolutely stable at zero temperature. The widest literature is devoted to the quantum mechanics of two-electron atomic shell (the main part of this literature is reviewed and discussed in the classical books $[9,10]$, for recent publications see also reviews Refs. 11, 12). From the formally point of view the Schrödinger equation for two mutually repulsive electrons in the central attractive Coulomb field of the infinite heavy nucleus is a linear sixvariable differential equation of second order in partial differentials. An exact analytical solution of this equation is still unknown [12], so that this problem was treated by a number of approximate approaches. Since the initial paper of Hylleraas [13] the Ritz variational approach based on artificial trial functions of different kind [14-22] predominates in the problem of helium states. It should be noted that all these results are the conditional variational solutions with reduced (as compared with initial six-coordinate 
statement) number of spatial variables, so that they are approximate solutions whose accuracy should be estimated by independent methods. The energy of the helium atom ground state was also estimated by various approximate methods as perturbation study with 1/Z-expansion [23-25], semiclassical approach [26,27] and Hartree-Fock approximation [9,10,28-34].

Mathematically, the solution of a linear differential equation can be built unambiguously as an expansion over a complete orthonormalized basis. As the corresponding basis it should be used any complete set of eigenfunctions belonging to a suitable Sturm-Liouville problem [35,36]. Thus, the initial problem reduces to an algebraic problem on eigenvalues and eigenvectors of an Hermitian matrix. The algebraic problem can be solved by direct diagonalization of the obtained matrix, and it is the essence of exact diagonalization method. In this paper we apply this method to the spinless helium atom in an external electric field.

In Sec. 2, we present the statement of the problem. In Sec. 3, we describe the method to solve the problem in general. Section 4 contains the diagonalization for the case of zero field with basis of dimensionality $N=25$ to illustrate the structure and classification of eigenstates of free helium atom. In Sec. 5 we show the field dependences of lowlying levels and polarizability of the ground state.

\section{Statement of the problem}

Helium electronic shell consists of two electrons which we denote as "a” and "b" particle, and all the values belonging to corresponding particle will be labeled by the corresponding index. In the spherical coordinate system with origin at immobile nucleus the radius-vectors of particles are $\mathbf{r}_{a}=\left\{r_{a}, \vartheta_{a}, \varphi_{a}\right\}, \mathbf{r}_{b}=\left\{r_{b}, \vartheta_{b}, \varphi_{b}\right\}$, and the Hamiltonian of the spinless helium shell $\left({ }^{4} \mathrm{He}\right)$ under external stationary and uniform electric field $\mathbf{E}$ can be written using the standard Hartree units $[9,37]$ in the form

$$
\hat{H}=\hat{H}^{(0)}+\mathbf{E}\left(\mathbf{r}_{a}+\mathbf{r}_{b}\right) .
$$

Here $\hat{H}^{(0)}$ is the Hamiltonian of the free (at $\mathbf{E}=0$ ) helium shell,

$$
\hat{H}^{(0)}=\hat{H}^{(a)}+\hat{H}^{(b)}+\hat{H}_{\text {int }},
$$

where one-particle Hamiltonians $\hat{H}^{(a)}$ and $\hat{H}^{(b)}$ are

$$
\hat{H}^{(a)}=-\frac{1}{2} \Delta_{a}+\frac{Z_{a} Z_{0}}{r_{a}}, \quad \hat{H}^{(b)}=-\frac{1}{2} \Delta_{b}+\frac{Z_{b} Z_{0}}{r_{b}},
$$

and the interaction term, $\hat{H}_{\mathrm{int}}$, is

$$
\hat{H}_{\mathrm{int}}\left(\mathbf{r}_{a}, \mathbf{r}_{b}\right)=\frac{Z_{a} Z_{b}}{\left|\mathbf{r}_{a}-\mathbf{r}_{b}\right|}
$$

The nucleus charge is $Z_{0}=+2$ and particle charges are $Z_{a}=Z_{b}=-1$.
An important property of the free atom (at $\mathbf{E}=0$ ) is that the square of the total angular momentum of its shell,

$$
\hat{L}^{2}=\left(\hat{\mathbf{L}}^{(a)}+\hat{\mathbf{L}}^{(b)}\right)^{2}
$$

and z-projection of the shell angular momentum,

$$
\hat{L}_{z}=-i\left(\frac{\partial}{\partial \varphi_{a}}+\frac{\partial}{\partial \varphi_{b}}\right),
$$

obviously commutate with Hamiltonian (2) [38]. Thus, the both dynamical variables are integrals of motion for fieldfree problem which means conservation both of the total angular momentum and its z-projection on an arbitrary quantization axis. However, these variables have somewhat different meaning. To clarify this statement we introduce, as the temporary variables, new azimuthal coordinates

$$
\Phi_{+}=\varphi_{a}+\varphi_{b}, \quad \Phi_{-}=\varphi_{a}-\varphi_{b}, \quad \hat{L}_{z}=-2 i \frac{\partial}{\partial \Phi_{+}},
$$

and rewrite the angular-dependent part of the interaction Hamiltonian, $\hat{H}_{\text {int }}$, in the form

$$
\begin{gathered}
\hat{H}_{\mathrm{int}}\left(r_{a}, r_{b}, \vartheta_{a}, \vartheta_{b}, \Phi_{-}\right)=\left\{r_{a}^{2}+r_{b}^{2}-\right. \\
\left.-2 r_{a} r_{b}\left(\cos \vartheta_{a} \cos \vartheta_{b}+\sin \vartheta_{a} \sin \vartheta_{b} \cos \Phi_{-}\right)\right\}^{-1 / 2} .
\end{gathered}
$$

It is seen that $\Phi_{+}$is a cyclic variable because the total Hamiltonian $\hat{H}$ does not depend on $\Phi_{+}$, so that its conjugate dynamical variable $\hat{L}_{z}$ is an integral of motion. Eigenvalues of this operator,

$$
M=m_{a}+m_{b}=0, \pm 1, \pm 2 \ldots
$$

(here $m_{a}$ and $m_{b}$ are magnetic quantum numbers of individual electrons) serve as good quantum numbers of the general problem. It means that every pure eigenstate of the Hamiltonian, $\hat{H}_{0}$, can be represented as infinite superposition of pair compositions of single particle wave functions with arbitrary $m_{a}, m_{b}$, but only under condition (6). This property will be used below for classification of the twoelectron shell states.

\section{Exact diagonalization method}

Mathematically, the spinless Schrödinger equation

$$
\hat{H} \Psi\left(\mathbf{r}_{a}, \mathbf{r}_{b}\right)=\mathcal{E} \Psi\left(\mathbf{r}_{a}, \mathbf{r}_{b}\right)
$$

is the linear partial differential equation of second order with six spatial variables $\mathbf{r}_{a}, \mathbf{r}_{b}$, and the variables can not be separated due to the special form of $\hat{H}_{\text {int }}$. An exact analytical solution of the two-particle equation (1) even at $\mathbf{E}=0$ is still unknown [12], let alone the case of nonzero external fields. Up to now the problem of free helium atom was analyzed with different approximate methods, among them in the most common use are direct Ritz variational approach [14-22], self-consistent (Hartree-Fock) approximation $[9,10,28-34]$ and perturbation theory $[9,10]$. An ef- 
fect of external electric fields was discussed exclusively within framework of standard perturbation theory with computation methods based on a certain variational procedure $[39,40]$. Here we shall build the formally exact solution of the problem Eq. (1) by exact diagonalization method which in our case is, in fact, standard Fourier procedure of mathematical physics [35,36]. It provides derivation of the solution in the form of an expansion over a basis built of the selected complete set of orthonormalized functions.

Below, we consider only bound states of two-particle Hamiltonian (1) with discrete spectrum $\mathcal{E}<0$. Let us find the exact solution $\Psi\left(\mathbf{r}_{a}, \mathbf{r}_{b}\right)$ of the linear partial differential equation (1) as an expansion [35,36]

$$
\Psi\left(\mathbf{r}_{a}, \mathbf{r}_{b}\right)=\sum_{p=1}^{\infty} c_{p} u_{p}\left(\mathbf{r}_{a}, \mathbf{r}_{b}\right)
$$

over a complete set $\left\{u_{p}\left(\mathbf{r}_{a}, \mathbf{r}_{b}\right)\right\}$ of functions orthonormalized with standard condition

$$
\left\langle u_{p} \mid u_{q}\right\rangle=\int d^{3} \mathbf{r}_{a} d^{3} \mathbf{r}_{b} u_{p}^{+}\left(\mathbf{r}_{a}, \mathbf{r}_{b}\right) u_{q}\left(\mathbf{r}_{a}, \mathbf{r}_{b}\right)=\delta_{p q},
$$

where $\delta_{p q}$ is Kronecker delta. The solution should satisfy the natural reciprocity relations, $\Psi\left(\mathbf{r}_{a}, \mathbf{r}_{b}\right)= \pm \Psi\left(\mathbf{r}_{b}, \mathbf{r}_{a}\right)$, which means the invariance relative to spatial permutations of the equivalent shell electrons. To determine the solution of Eq. (1) unambiguously, the coefficients $a_{p}$ have to be obtained by any reasonable procedure. If the elements of the basis $\left\{u_{p}\left(\mathbf{r}_{a}, \mathbf{r}_{b}\right)\right\}$ are not eigenfunctions of the Hamiltonian $\hat{H}$ then determination of the coefficients $c_{p}$ by substitution of Eq. (8) into Eq. (1) with regard to condition (9) leads to infinite system of linear algebraic equations principally coupled each other,

$$
\sum_{q=1}^{\infty}\left(H_{p q}-\mathcal{E} \delta_{p q}\right) c_{q}=0
$$

where

$H_{p q}=\left\langle u_{p}|\hat{H}| u_{q}\right\rangle=\int d^{3} \mathbf{r}_{a} d^{3} \mathbf{r}_{b} u_{p}^{+}\left(\mathbf{r}_{a}, \mathbf{r}_{b}\right) \hat{H} u_{q}\left(\mathbf{r}_{a}, \mathbf{r}_{b}\right)$.

Thus, the problem is reduced to standard algebraic eigenvalue problem with infinite Hermitian matrix $H_{p q}$.

The best way to solve the problem (10) is diagonalization of $H_{p q}$ by corresponding unitary transformation [41,42],

$$
\sum_{p=1}^{\infty} \sum_{q=1}^{\infty} \mathbf{S}_{i p}^{-1} H_{p q} \mathbf{S}_{q j}=\operatorname{diag}_{i j}\left\{\varepsilon_{1}, \varepsilon_{2}, \ldots, \varepsilon_{k} \ldots\right\},
$$

where

$$
\sum_{p=1}^{\infty} \mathbf{S}_{i p}^{-1} \mathbf{S}_{p j}=\delta_{i j} ; \quad \text { Det } \hat{\mathbf{S}}=1 .
$$

The eigenvalues $\varepsilon_{1} \leq \varepsilon_{2} \leq \ldots \leq \varepsilon_{k} \leq \ldots$ are the spectrum of the problem (10), including degenerated states. The $j$-column of the unitary matrix $\mathbf{S}_{p j}$ consists of the components of the infinite-dimensional eigenvector $\left|e_{p}^{(j)}\right\rangle$ of the matrix $H_{p q}$ belonging to eigenvalue $\varepsilon_{j}$,

$$
\sum_{q=1}^{\infty} H_{p q} e_{q}^{(j)}=\varepsilon_{j} e_{p}^{(j)}
$$

The eigenvectors $\left|e_{p}^{(j)}\right\rangle$ are naturally orthonormalized by definition,

$$
\sum_{p=1}^{\infty} e_{p}^{(j)} e_{p}^{(k)}=\delta_{j k}
$$

The components of eigenvectors realize the representation of corresponding eigenstates in the initial basis $\left\{u_{p}\left(\mathbf{r}_{a}, \mathbf{r}_{b}\right)\right\}$,

$$
\Psi_{(j)}\left(\mathbf{r}_{a}, \mathbf{r}_{b}\right)=\sum_{p=1}^{\infty} e_{p}^{(j)} u_{p}\left(\mathbf{r}_{a}, \mathbf{r}_{b}\right)
$$

It is easy to check that Eq. (16) is an eigenvector of Hamiltonian $\hat{H}$. As a result, the solution of Eq. (1) is reduced to algebraic problem of eigenstates (14) with infinite Hermitian matrix $H_{p q}$. A problem of such kind can be solved with any desirable accuracy by different numerical methods [41,42].

Practically, the solution of the infinite algebraic system Eqs. (10), (12) is usually built by successive approximations $[41,43]$. Let us suppose that the system is obtained using the truncated functional basis $\left\{u_{p}^{(N)}\left(\mathbf{r}_{a}, \mathbf{r}_{b}\right)\right\}$ consisting of first $N$ functions of the complete basis $\left\{u_{p}\left(\mathbf{r}_{a}, \mathbf{r}_{b}\right)\right\}$, so that

$$
\Psi^{(N)}\left(\mathbf{r}_{a}, \mathbf{r}_{b}\right)=\sum_{p=1}^{N} c_{p}^{(N)} u_{p}^{(N)}\left(\mathbf{r}_{a}, \mathbf{r}_{b}\right)
$$

The corresponding algebraic system based on $N \times N$ Hermitian submatrix $H_{p q}^{(N)}$ of the complete matrix $H_{p q}$ produces $N$ eigenvalues, $\varepsilon_{1}^{(N)}, \varepsilon_{2}^{(N)}, \ldots, \varepsilon_{N}^{(N)}$, and eigenvectors of dimensionality $N$ [41]. This system can be considered as $N$-approximate solution of the initial infinite-dimensional problem of eigenstates. To prove the convergence of the procedure we calculate $(N+1)$-approximate solution based on first $(N+1)$ basis functions, $\left\{u_{p}\left(\mathbf{r}_{a}, \mathbf{r}_{b}\right)\right\}_{N+1}$, which generate an $(N+1) \times(N+1)$ Hermitian matrix, $H_{p q}^{(N+1)}$. The eigenvalues of this $(N+1)$-approximation are $\varepsilon_{1}^{(N+1)}, \varepsilon_{2}^{(N+1)}, \ldots, \varepsilon_{N+1}^{(N+1)}$. The described process leads to the following chain of inequalities for successive approximate eigenvalues [41,43,44]

$$
\left|\varepsilon_{j}^{(N)}\right| \leq\left|\varepsilon_{j}^{(N+1)}\right| \leq\left|\varepsilon_{j}\right|,
$$

where $j=1,2, \ldots, N$, and $\varepsilon_{j}$ is an exact $j$-eigenvalue, obtained as a limit at $N \rightarrow \infty$. It means monotonic convergence of the described procedure. The rigorous prove of the convergence demands more detailed mathematical treatment of the asymptotic behavior of the matrix ele- 
ments $H_{p q}$ at $p, q \rightarrow \infty$. The analysis of corresponding criteria [41,44] is far from goals of the present paper, it will be a matter of special research. Eigenvalues and eigenvectors can be found for each submatrix $H_{p q}^{(N)}$ and, consequently, the main problem can be solved practically with any desirable accuracy if the elements $H_{p q}^{(N)}$ are built exactly at arbitrary $N$. The described approach reduces the functional problem with differential equation to an infinite algebraic eigenvalue problem whose spectrum and the functional representation Eq. (16) of eigenvectors in the limit $N \rightarrow \infty$ coincides exactly with the spectrum and eigenstates of the original Hamiltonian. Eigenvectors of the algebraic problem give representations for eigenfunctions of original Hamiltonian in the basis $\left\{u_{p}\left(\mathbf{r}_{a}, \mathbf{r}_{b}\right)\right\}$.

\section{Eigenstates of two-electron shell at $\mathrm{E}=0$}

Here we start from the solution for free helium atom without external fields. We produce the spectrum and wave functions of isolated helium atom (at $\mathbf{E}=0$ ) with Hamiltonian (2). It will make us below to explain corresponding field dependences for arbitrary fields $\mathbf{E} \neq 0$.

For our goals it is naturally to select the basis $\left\{u_{p}\left(\mathbf{r}_{a}, \mathbf{r}_{b}\right)\right\}$ as every possible direct products,

$$
\begin{aligned}
u_{p}\left(\mathbf{r}_{a}, \mathbf{r}_{b}\right)=\psi_{n_{a} l_{a} m_{a}}\left(\alpha r_{a}, \vartheta_{a}, \varphi_{a}\right) \psi_{n_{b} l_{b} m_{b}}\left(\alpha r_{b}, \vartheta_{b}, \varphi_{b}\right) \equiv \\
\equiv\left|\begin{array}{ccc}
n_{a} & l_{a} & m_{a} \\
n_{b} & l_{b} & m_{b}
\end{array}\right|
\end{aligned}
$$

of single-particle hydrogen-like functions [10,46],

$$
\psi_{n l m}(\alpha \mathbf{r})=R_{n l}(\alpha r) Y_{l m}(\vartheta, \varphi) \equiv|n l m\rangle,
$$

where $Y_{I m}(\vartheta, \varphi)$ are spherical harmonics in standard determination [10,37], and radial function $R_{n l}(\alpha r)$ depends on radial coordinate renormalized by scale parameter $\alpha>0$,

$$
\begin{aligned}
R_{n l}(\alpha r) & =-\frac{2 \alpha^{3 / 2}}{n^{2}} \sqrt{\frac{(n-l-1) !}{[(n+l) !]^{3}}}\left(\frac{2 \alpha r}{n}\right)^{l} \times \\
\times & L_{n+l}^{2 l+1}\left(\frac{2 \alpha r}{n}\right) \exp \left(-\frac{\alpha r}{n}\right),
\end{aligned}
$$

$$
L_{n}^{m}(x)=(-1)^{m}(n !)^{2} \sum_{k=0}^{n-m} \frac{(-x)^{k}}{k !(m+k) !(n-m-k) !}
$$

Note, that functions $R_{n l}$ of the set Eq. (21) are orthonormalized at any real positive $\alpha>0$. The case $\alpha=1$ in single-particle function corresponds to the simple hydrogen atom. For two-electron helium shell we have $\alpha=Z_{0}-\sigma$, where $0<\sigma<1$ is the screening parameter $[9,37,45]$. The presence of the screening parameter $\sigma$ means that each of the electrons moves in the nucleus field renormalized due to influence of another electron. Physically, this fact can be considered as an effective account of Hartree's self-consistency.

Each basis function number

$$
p \rightarrow\left\{\begin{array}{lll}
n_{a} & l_{a} & m_{a} \\
n_{b} & l_{b} & m_{b}
\end{array}\right\}
$$

is determined in univocal correspondence to a double set of single-particle hydrogen-like quantum numbers. The complete basis must include all functions $u_{n_{a} l_{a} m_{a}}\left(\mathbf{r}_{a}\right)$ and $u_{n_{b} l_{b} m_{b}}\left(\mathbf{r}_{b}\right)$ corresponding to all degenerate states with different $m_{a}$ and $m_{b}$ at given $n_{a}, n_{b}, l_{a}, l_{b}$. The complete basis must contain all $m$-dependent functions because azimuthal variables are connected directly with true quantum number $M$ of the general problem. Each function (19) is the eigenfunction of the $L_{z}$-operator (see Eq. (5)) with eigenvalue $M=m_{a}+m_{b}$. Consequently, all eigenstates of the Hamiltonian (2) which are linear combinations of the basis elements (19) with different $m_{a}, m_{b}$ will be evidently eigenfunctions of $L_{z}$ corresponding to determined $M$. Thus, the total space of the basis functions is naturally separated on subspaces from basis elements belonging to the determined $M=m_{a}+m_{b}$. This property is principally important for rigorous classification of two-particle states.

As a result, we will find the solution of the problem

$$
\hat{H}^{(0)} \Psi\left(\mathbf{r}_{a}, \mathbf{r}_{b}\right)=\mathcal{E}^{(0)} \Psi\left(\mathbf{r}_{a}, \mathbf{r}_{b}\right)
$$

in the form Eq. (8) with the basis (19). Matrix elements of the Hamiltonian $H^{(0)}$ on the basis (19) are (cmp. Ref. 37)

where $L_{n}^{m}(x)$ are Laguerre polynomials,

$$
H_{p q}^{(0)}=\left\langle u_{p}\left|H^{(0)}\right| u_{q}\right\rangle=-\frac{\alpha^{2}}{2}\left(\frac{1}{n_{a}^{2}}+\frac{1}{n_{b}^{2}}\right) \delta_{p q}-\sigma\left\langle u_{p}\left|\frac{1}{r_{a}}+\frac{1}{r_{b}}\right| u_{q}\right\rangle+\left\langle u_{p}\left|\frac{1}{\left|\mathbf{r}_{a}-\mathbf{r}_{b}\right|}\right| u_{q}\right\rangle \quad\left(n_{a}, n_{b}=1,2, \ldots\right)
$$

The matrix elements of interelectron repulsion $\hat{H}_{\text {int }}$ can be obtained through cumbersome, but elementary integrals,

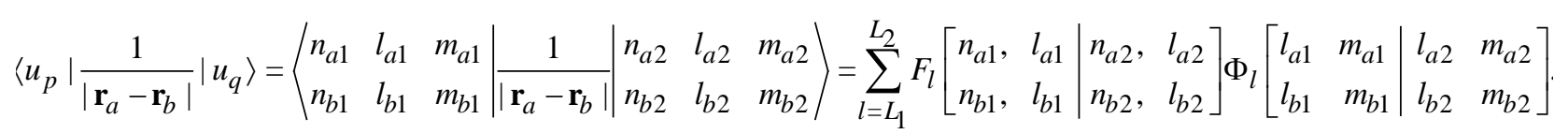


Here

$$
\begin{aligned}
& \Phi_{l}\left[\begin{array}{cc|cc}
l_{a 1} & m_{a 1} & l_{a 2} & m_{a 2} \\
l_{b 1} & m_{b 1} & l_{b 2} & m_{b 2}
\end{array}\right]=\sqrt{\left(2 l_{a 1}+1\right)\left(2 l_{a 2}+1\right)\left(2 l_{b 1}+1\right)\left(2 l_{b 2}+1\right)}\left(\begin{array}{ccc}
l_{a 1} & l_{a 2} & l \\
0 & 0 & 0
\end{array}\right)\left(\begin{array}{ccc}
l_{b 1} & l_{b 2} & l \\
0 & 0 & 0
\end{array}\right) \times \\
& \times \sum_{m=-l}^{l}(-1)^{m+M}\left(\begin{array}{ccc}
l_{a 1} & l_{a 2} & l \\
-m_{a 1} & m_{a 2} & -m
\end{array}\right)\left(\begin{array}{ccc}
l_{b 1} & l_{b 2} & l \\
-m_{b 1} & m_{b 2} & m
\end{array}\right),
\end{aligned}
$$

where $\left(\begin{array}{lll}a & b & c \\ d & e & f\end{array}\right)$ are Wigner $3 j$-symbols [47],

$$
M=m_{a 1}+m_{b 1}=m_{a 2}+m_{b 2}=0, \pm 1, \pm 2, \ldots
$$

is the shell quantum number (see Eq. (6)),

$L_{1}=\max \left\{\left|l_{a 1}-l_{a 2}\right|,\left|l_{b 1}-l_{b 2}\right|\right\}, L_{2}=\min \left\{l_{a 1}+l_{a 2}, l_{b 1}+l_{b 2}\right\}$ (max $\{a, b\}$ or $\min \{a, b\}$ means maximal or minimal value of two comparable integers, respectively). The Wigner symbol $\left(\begin{array}{lll}l_{1} & l_{2} & l \\ 0 & 0 & 0\end{array}\right) \neq 0$ only if $l_{1}+l_{2}+l=2 s \geq 0$ is even integer and $\left(\begin{array}{lll}a & b & c \\ d & e & f\end{array}\right) \neq 0$ only if $d+e+f=0$ (see Refs. 46-48). Thus, nontrivial matrix elements exist at the compatibility of the conditions $-m_{a 1}+m_{a 2}-m=0$ and $-m_{b 1}+m_{b 2}+m=0$, as well as the conditions $l_{a 1}+l_{a 2}+l=2 p$ and $l_{b 1}+l_{b 2}+l=2 q$, $p$ and $q$ are positive integers. The first pair of the equalities reduces to the identity $M \equiv M$, whereas the second pair gives $\left(l_{a 1}-l_{b 1}\right)+\left(l_{a 2}-l_{b 2}\right)=2(p-q)$ which means that both of $l_{a 1}-l_{b 1}$ and $l_{a 2}-l_{b 2}$ are odd or even simultaneously (zero is considered as the even integer). Consequently, at any determined $M$ the total space of solutions separates onto $2(2|M|+1)$ subspaces corresponding to each $0 \leq\left|M^{\prime}\right| \leq|M|$ and divided additionally at any determined $M^{\prime}$ on two subspaces with a certain parity of $\left|l_{a}-l_{b}\right|$. Function $F_{l}$ as double integral over radial variables can be represented in the form of multiple finite numerical sums (see Appendix 1).
Matrix elements of the nucleus-electron interaction are

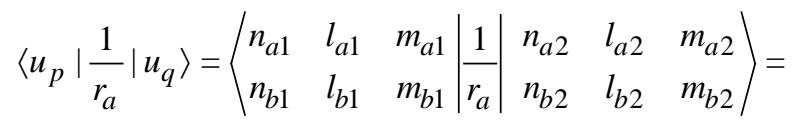

$$
\begin{aligned}
& =\delta_{n_{b 1} n_{b 2}} \delta_{l_{b 1} l_{b 2}} \delta_{m_{b 1} m_{b 2}} \delta_{l_{a 1} l_{a 2}} \delta_{m_{a 1} m_{a 2}} \times \\
& \times \mathcal{I}_{-1}\left(n_{a 1}, l_{a 1} \mid n_{a 2}, l_{a 2}\right), \\
& \left\langle u_{p}\left|\frac{1}{r_{b}}\right| u_{q}\right\rangle=\left\langle\begin{array}{lll}
n_{a 1} & l_{a 1} & m_{a 1} \\
n_{b 1} & l_{b 1} & m_{b 1}
\end{array}\left|\frac{1}{r_{b}}\right| \begin{array}{ccc}
n_{a 2} & l_{a 2} & m_{a 2} \\
n_{b 2} & l_{b 2} & m_{b 2}
\end{array}\right\rangle= \\
& =\delta_{n_{a 1} n_{a 2}} \delta_{l_{a 1} l_{a 2}} \delta_{m_{a 1} m_{a 2}} \delta_{l_{b 1} l_{b 2}} \delta_{m_{b 1} m_{b 2}} \times \\
& \times \mathcal{I}_{-1}\left(n_{b 1}, l_{b 1} \mid n_{b 2}, l_{b 2}\right),
\end{aligned}
$$

where integral $\mathcal{I}_{S}$ is presented in Appendix 2. Thus, all matrix elements (26), (28), (29) can be obtained as observable analytical expressions and calculated with any desirable accuracy.

The total angular momentum $\hat{\mathbf{L}}=\hat{\mathbf{L}}^{(a)}+\hat{\mathbf{L}}^{(b)}$ of the shell is a dynamic variable which conserves due to central symmetry of the two-electron system relative to center on the nucleus. The angular and translational variables can not be separated in the Schrödinger equation (24), so that there are not a simple quantization rule for $\hat{L}^{2}$, as we have for $\hat{L}_{z}$. However, the matrix elements $\left\langle u_{p}\left|\hat{L}^{2}\right| u_{q}\right\rangle$ can be calculated easily, so that we have an average value of this operator in each eigenstate with number $j$,

$$
\begin{gathered}
\left\langle\Psi_{(j)}\left(\mathbf{r}_{a}, \mathbf{r}_{b}\right)\left|\hat{L}^{2}\right| \Psi_{(j)}\left(\mathbf{r}_{a}, \mathbf{r}_{b}\right)\right\rangle=\sum_{p=1}^{\infty} \sum_{q=1}^{\infty} e_{p}^{(j)} e_{q}^{(j)}\left\langle u_{p}\left(\mathbf{r}_{a}, \mathbf{r}_{b}\right)\left|\hat{L}^{2}\right| u_{q}\left(\mathbf{r}_{a}, \mathbf{r}_{b}\right)\right\rangle= \\
=\sum_{p=1}^{\infty} \sum_{q=1}^{\infty} e_{p}^{(j)} e_{q}^{(j)} \delta_{n_{a 1}, n_{a 2}} \delta_{n_{b 1}, n_{b 2}} \delta_{l_{a 1}, l_{a 2}} \delta_{l_{b 1}, l_{b 2}}\left\{\left[l_{a 2}\left(l_{a 2}+1\right)+l_{b 2}\left(l_{b 2}+1\right)+2 m_{a 2} m_{b 2}\right] \delta_{m_{a 1}, m_{a 2}} \delta_{m_{b 1}, m_{b 2}}+\right. \\
+\sqrt{l_{a 2}\left(l_{a 2}+1\right)-m_{a 2}\left(m_{a 2}+1\right)} \sqrt{l_{b 2}\left(l_{b 2}+1\right)-m_{b 2}\left(m_{b 2}-1\right)} \delta_{m_{a 1}, m_{a 2}+1} \delta_{m_{b 1}, m_{b 2}-1}+ \\
\left.+\sqrt{l_{a 2}\left(l_{a 2}+1\right)-m_{a 2}\left(m_{a 2}-1\right)} \sqrt{l_{b 2}\left(l_{b 2}+1\right)-m_{b 2}\left(m_{b 2}+1\right)} \delta_{m_{a 1}, m_{a 2}-1} \delta_{m_{b 1}, m_{b 2}+1}\right\}
\end{gathered}
$$

with correspondence between $p, q$ and others indexes given by Eq. (23).

After diagonalization $H_{p q}^{(0)}(25)$ we have spectrum and eigenstates of the two-electron atom. In our calculations, as usual, $\alpha$ will be considered as an additional nonlinear variational parameter (cmp. with Refs. 9, 37, 45) which is determined from direct optimization of the ground state energy $\varepsilon_{1}^{(0)}(\alpha)$ obtained with truncaded basis of dimensionality $N$,

$$
\frac{\partial \varepsilon_{1}^{(0)}(\alpha)}{\partial \alpha}=0
$$

In our statement, it can be proved that $\alpha \rightarrow Z_{0}$ (or $\sigma \rightarrow 0$ ) with $N \rightarrow \infty$, as the approximate solution tends to its exact form. Of course, the problem can contain a set of nonlinear parameters (see, for example, Refs. 21, 22) with corresponding modification in conditions for their optimization. 
Here we use the basis set formed by pair combinations of five single-particle hydrogen-like states corresponding to $n=1,2$. Nomination of this two-particle basis functions is given in Appendix 3. As a result, we obtain the Hermitian matrix $H_{p q}$ of the $25 \times 25$ dimensionality with spectrum $\varepsilon_{j}^{(0)}(1 \leq j \leq 25)$ and eigenvectors $e_{p}^{(j)}$. The diagonalization of this matrix is fulfilled by Jacobi method [49] with an absolute accuracy (for energy levels and eigenvectors orthonormality) of $10^{-13}$. The spectrum of the problem (at screening constant $\sigma=0.23231$ ) is shown in Table 1 . The wave function of state with number $j$ is

$$
\Psi_{(j)}\left(\mathbf{r}_{a}, \mathbf{r}_{b}\right)=\sum_{p=1}^{25} e_{p}^{(j)} u_{p}\left(\mathbf{r}_{a}, \mathbf{r}_{b}\right)
$$

Table 1. Helium spectrum in the truncated basis $N=25$

\begin{tabular}{c|c|c|c|c|c|c}
\hline \hline Level & $\varepsilon_{p}$, a.u. & $M$ & $l_{a}$ & $l_{b}$ & $\left\langle\hat{\mathbf{L}}^{2}\right\rangle$ & $\begin{array}{c}\text { Symmetry } \\
\text { of state }\end{array}$ \\
\hline \hline$\varepsilon_{1}^{(0)}$ & -2.85688 & 0 & 0 & 0 & 0 & $s$ \\
$\varepsilon_{2}^{(0)}$ & -2.05239 & 0 & 0 & 0 & 0 & $s$ \\
$\varepsilon_{3}^{(0)}$ & -2.13341 & 0 & 0 & 0 & 0 & $a$ \\
$\varepsilon_{4}^{(0)}$ & -2.06874 & 0 & 0 & 1 & 2 & $a$ \\
$\varepsilon_{5}^{(0)}$ & -2.01338 & 0 & 1 & 0 & 2 & $s$ \\
$\varepsilon_{6}^{(0)}$ & -2.06882 & -1 & 0 & 1 & 2 & $a$ \\
$\varepsilon_{7}^{(0)}$ & -2.01314 & -1 & 1 & 0 & 2 & $s$ \\
$\varepsilon_{8}^{(0)}$ & -2.06882 & 1 & 0 & 1 & 2 & $a$ \\
$\varepsilon_{9}^{(0)}$ & -2.01314 & 1 & 1 & 0 & 2 & $s$ \\
$\varepsilon_{10}^{(0)}$ & -0.76609 & 0 & 0 & 0 & 0 & $s$ \\
$\varepsilon_{11}^{(0)}$ & -0.64193 & 0 & 0 & 1 & 2 & $s$ \\
$\varepsilon_{12}^{(0)}$ & -0.74991 & 0 & 1 & 0 & 2 & $a$ \\
$\varepsilon_{13}^{(0)}$ & -0.59056 & -1 & 0 & 1 & 2 & $s$ \\
$\varepsilon_{14}^{(0)}$ & -0.80144 & -1 & 1 & 0 & 2 & $a$ \\
$\varepsilon_{15}^{(0)}$ & -0.59057 & 1 & 0 & 1 & 2 & $s$ \\
$\varepsilon_{16}^{(0)}$ & -0.80144 & 1 & 1 & 0 & 2 & $a$ \\
$\varepsilon_{17}^{(0)}$ & -0.54766 & 0 & 1 & 1 & 0 & $s$ \\
$\varepsilon_{18}^{(0)}$ & -0.69679 & -1 & 1 & 1 & 2 & $a$ \\
$\varepsilon_{19}^{(0)}$ & -0.65963 & -1 & 1 & 1 & 6 & $s$ \\
$\varepsilon_{25}^{(0)}$ & -0.69679 & 1 & 1 & 1 & 2 & $a$ \\
$\varepsilon_{21}^{(0)}$ & -0.65963 & 1 & 1 & 1 & 6 & $s$ \\
$\varepsilon_{22}^{(0)}$ & -0.65963 & -2 & 1 & 1 & 6 & $s$ \\
$\varepsilon_{23}^{(0)}$ & -0.65963 & 0 & 1 & 1 & 6 & $s$ \\
\hline
\end{tabular}

It can be seen that total space of eigenstates decomposes to $2(2|M|+1)$ independent subspaces corresponding to each quantum number $0 \leq|M| \leq\left(l_{a}+l_{b}\right)$. The ground state $\Psi_{(1)}\left(\mathbf{r}_{a}, \mathbf{r}_{b}\right)$ (with $\varepsilon_{1}^{(0)}=-2.85688285585356$ ) is composed of basis functions $\psi_{p}\left(\mathbf{r}_{a}, \mathbf{r}_{b}\right)$ satisfying the condition of quantum number $M=m_{a}+m_{b}=0$ at $l_{a}=l_{b}$,

$$
\begin{gathered}
\Psi_{(1)}\left(\mathbf{r}_{a}, \mathbf{r}_{b}\right)=0.91428 u_{1}\left(\mathbf{r}_{a}, \mathbf{r}_{b}\right)-0.0892\left[u_{2}\left(\mathbf{r}_{a}, \mathbf{r}_{b}\right)+\right. \\
\left.+u_{3}\left(\mathbf{r}_{a}, \mathbf{r}_{b}\right)\right]-0.0223 u_{10}\left(\mathbf{r}_{a}, \mathbf{r}_{b}\right)-0.0145 u_{17}\left(\mathbf{r}_{a}, \mathbf{r}_{b}\right)+ \\
+0.0145\left[u_{23}\left(\mathbf{r}_{a}, \mathbf{r}_{b}\right)+u_{24}\left(\mathbf{r}_{a}, \mathbf{r}_{b}\right)\right] .
\end{gathered}
$$

Within the system of two fermions in the central field of spinless nucleus (atom ${ }^{4} \mathrm{He}$ ) it should be completed by antisymmetric singlet spin function [37]. The average square of the shell angular momentum Eq. (30) in the ground states is equal to zero (see Table 1) despite the eigenstate (33) is a superposition of basis elements with $l, m \neq 0$. Thus, the ground state $\varepsilon_{1}^{(0)}$ is the spatially symmetric level of $1 s 1 s^{1} S_{0}$ nomenclature (para-helium) [50].

The second on the energy scale is the spatially antisymmetric level $\varepsilon_{3}^{(0)}=-2.13340673120655$ with wave function

$$
\Psi_{(3)}\left(\mathbf{r}_{a}, \mathbf{r}_{b}\right)=-\frac{1}{\sqrt{2}}\left[u_{2}\left(\mathbf{r}_{a}, \mathbf{r}_{b}\right)-u_{3}\left(\mathbf{r}_{a}, \mathbf{r}_{b}\right)\right] .
$$

In the case of spinless ${ }^{4} \mathrm{He}$ nucleus it should completed by the triplet spin function [37], and it has to be nominated as the $1 s 2 s^{3} S_{1}$ state (the ground state of ortho-helium) [50]. The third level, $\varepsilon_{2}^{(0)}=-2.05239223447308$, is spatially symmetric state $1 s 2 s^{1} S_{0}$ with

$$
\begin{gathered}
\Psi_{(2)}\left(\mathbf{r}_{a}, \mathbf{r}_{b}\right)=0.12818 u_{1}\left(\mathbf{r}_{a}, \mathbf{r}_{b}\right)+0.6992\left[u_{2}\left(\mathbf{r}_{a}, \mathbf{r}_{b}\right)+\right. \\
\left.+u_{3}\left(\mathbf{r}_{a}, \mathbf{r}_{b}\right)\right]+0.07092 u_{10}\left(\mathbf{r}_{a}, \mathbf{r}_{b}\right)+0.0167 u_{17}\left(\mathbf{r}_{a}, \mathbf{r}_{b}\right)- \\
\quad-0.0167\left[u_{23}\left(\mathbf{r}_{a}, \mathbf{r}_{b}\right)+u_{24}\left(\mathbf{r}_{a}, \mathbf{r}_{b}\right)\right] .
\end{gathered}
$$

The most impressive result is that the next on the energy scale is spatially antisymmetric triplet $1 s 2 p^{3} P_{0,1,2}^{0}$ of levels $\varepsilon_{4}^{(0)}=-2.06874256496409$ and $\varepsilon_{6}^{(0)}=\varepsilon_{8}^{(0)}=-2.06874256496454$. Lower state is doubly degenerated, but in reality it is slightly splitted and the distance between upper and lower sublevels is higher due to spin-orbital interaction [50]. Three wave functions, $\Psi_{(4)}, \Psi_{(6)}$ and $\Psi_{(8)}$, are presented in Appendix 4. No more comparisons with experimental spectroscopic data could be made because the used basis is restricted by only single-particle states with $n_{a}, n_{b}=1,2$. As it can be seen, even without taking into account any possibly nonrelativistic and relativistic corrections [9] our theoretical calculations agree with experiment on a good quantitative level. Our approximate solution reproduces correctly the real nomenclature of low-lying states of the real helium shell [50]. 


\section{Two-electron shell under uniform static electric field}

In this section we apply the above-described procedure to the total Hamiltonian (1). Let us suppose that electric field $\mathbf{E}$ has the only component $E_{z}=E_{0}$. Then, in the basis of Sec. 4

$$
H_{p q}=H_{p q}^{(0))}+E_{0}\left\langle u_{p}\left|\left(z_{a}+z_{b}\right)\right| u_{q}\right\rangle
$$

where

$$
\begin{aligned}
& \left\langle u_{p}\left|\left(z_{a}+z_{b}\right)\right| u_{q}\right\rangle=\delta_{n_{b 1} n_{b 2}} \delta_{l_{b 1} l_{b 2}} \delta_{m_{a 1} m_{a 2}} \delta_{m_{b 1} m_{b 2}}\left[\sqrt{\frac{\left(l_{a 2}-m_{a 2}+1\right)\left(l_{a 2}+m_{a 2}+1\right)}{\left(2 l_{a 2}+1\right)\left(2 l_{a 2}+3\right)}} \delta_{l_{a 1} l_{a 2+1}}+\right. \\
& \left.+\sqrt{\frac{\left(l_{a 2}-m_{a 2}\right)\left(l_{a 2}+m_{a 2}\right)}{\left(2 l_{a 2}-1\right)\left(2 l_{a 2}+1\right)}} \delta_{l_{a 1} l_{a 2-1}}\right] \mathcal{I}_{1}\left(n_{a 1}, l_{a 1} \mid n_{a 2}, l_{a 2}\right)+ \\
& +\delta_{n_{a 1} n_{a 2}} \delta_{l_{a 1} l_{a 2}} \delta_{m_{b 1} m_{b 2}} \delta_{m_{a 1} m_{b a}}\left[\sqrt{\frac{\left(l_{b 2}-m_{b 2}+1\right)\left(l_{b 2}+m_{b 2}+1\right)}{\left(2 l_{b 2}+1\right)\left(2 l_{b 2}+3\right)}} \delta_{l_{b 1} l_{b 2+1}}+\right. \\
& \left.+\sqrt{\frac{\left(l_{b 2}-m_{b 2}\right)\left(l_{b 2}+m_{b 2}\right)}{\left(2 l_{b 2}-1\right)\left(2 l_{b 2}+1\right)}} \delta_{l_{b 1} l_{b 2-1}}\right] \mathcal{I}_{1}\left(n_{b 1}, l_{b 1} \mid n_{b 2}, l_{b 2}\right) .
\end{aligned}
$$

After diagonalization according to the procedure of Sec. 3 we have spectrum $\varepsilon_{j}\left(E_{0}\right)$ and eigenvectors with components $e_{p}^{(j)}\left(E_{0}\right)$. It is easy to prove that the spectrum $\varepsilon_{j}\left(E_{0}\right)$ does not depend on a mutual orientation of the field $\mathbf{E}$ and $Z$ axis. Indeed, let us suppose that $\Theta_{0}$ is an angle between $\mathbf{E}$ and $z$ direction. We can reduce the problem to abovedescribed when rotate the coordinate system on angle $\Theta_{0}$ to superposition of $z$ axis with $\mathbf{E}$. Under such an operation each single-particle basis function $|n l m\rangle$ transforms as a linear combination of functions $\left|n l m^{\prime}\right\rangle$ with different $-l \leq m^{\prime} \leq l$ [47]. As a result, although components of eigenvectors $e_{p}^{(j)}$ are functions of $\Theta_{0}$, both spectrum $\varepsilon_{j}$ and average values of dynamical variables are independent on the angle $\Theta_{0}$ between $z$ and $\mathbf{E}$. In this connection, we can put $\mathbf{E}=\left\{0,0, E_{0}\right\}$.

It should be noted that our consideration is not a perturbation, but exact diagonalization approach. In this connection we obtain results which are correct at arbitrary $E_{0}$.

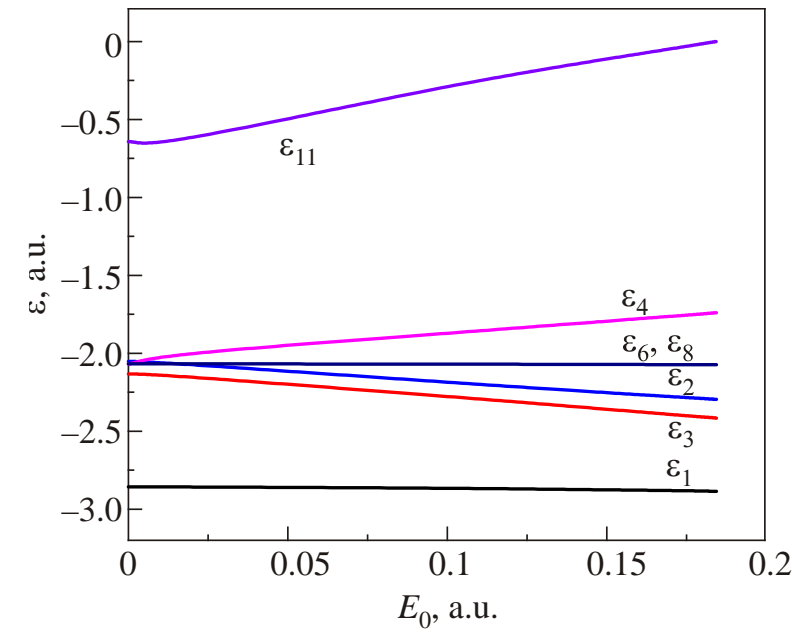

Fig. 1. Spectrum of ${ }^{4} \mathrm{He}$ lowest states vs external electric field. All values are given in atomic units.
Figure 1 shows the field dependences of the several lowest levels in helium spectrum. The ground-state energy $\varepsilon_{1}\left(E_{0}\right)$ $\left(1 s 1 s^{1} S_{0}\right)$ decreases slightly with $E_{0}$. Decrease in $\varepsilon_{3}\left(E_{0}\right)$ (the ground state $1 s 2 s^{3} S_{1}$ of ortho-helium) within the same field interval is more essential, so that the levels $\varepsilon_{1}\left(E_{0}\right)$ and $\varepsilon_{3}\left(E_{0}\right)$ approach each other when $E_{0}$ decreases. The behavior of $1 s 2 p^{3} P_{0,1,2}^{0}$ triplet levels is quite different. The highest level of the triplet increases with field, whereas two lowest levels remain degenerated and field-independent. For illustration, we show the behavior of $\varepsilon_{11}\left(E_{0}\right)$ level which rapidly decreases with $E_{0}$ and becomes zero at $E_{0}=0.18443425$. However, this fact can not be interpreted surely as the shell instability in view of finite dimensionality of the used basis. Anyway, we can conclude that helium spectrum could be sufficiently reconstructed under electric field of rather high intensity, comparable with intra-atomic fields. In reality, such fields can be produced by neighboring atom placed in the immediate vicinity of the actual shell.

Atomic polarizability $\alpha_{p}\left(E_{0}\right)$ in each $p$ state is determined by standard way [46],

$$
\left\langle d_{z}\right\rangle_{p}=\alpha_{p}\left(E_{0}\right) E_{0},
$$

through average dipole moment in the corresponding state,

$$
\left\langle d_{z}\right\rangle_{p}=\left\langle\Psi_{(p)}\left|\left(z_{a}+z_{b}\right)\right| \Psi_{(p)}\right\rangle,
$$

which can be calculated from Eq. (37). Field dependence of ${ }^{4}$ He polarizability $\alpha_{1}\left(E_{0}\right)$ in the ground state $\left(1 s 1 s^{1} S_{0}\right)$ is presented in Fig. 2. The calculated low field value $\alpha(0)=1.517$ is a bit higher than experimentally observed $\alpha=1.383$ [50], but this result should be considered as quite satisfactory in view of the fact that our calculation does not take into account neither nonrelativistic, nor relativistic corrections [9]. More essential is the fact that $\alpha_{1}\left(E_{0}\right)$ remains constant up to extremely high fields and demon- 


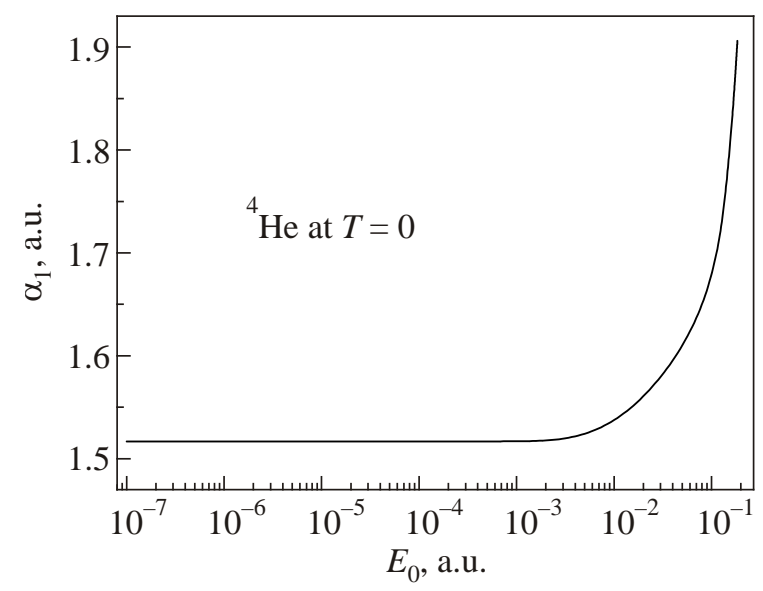

Fig. 2. Polarizability $\alpha_{1}$ of ${ }^{4} \mathrm{He}$ isolated atom in the ground state vs external electric field $E_{0}$ (atomic units).

strates an essential nonlinearity only at approximately $E_{0}>0.01$.

\section{Conclusions}

Our results are obtained within standard concept of mathematical physics [35] based on Fourier expansion for the solution of linear partial differential equation depending on six spatial variables (exact Schrödinger equation describing two-electron shell in the nucleus central field). Owing to exact analytical representation for any matrix elements of interaction Hamiltonian, the problem reduces to standard algebraic diagonalization of infinite Hermitian matrix which gives the spectrum and eigenvectors (wave functions of eigenstates) with any desirable accuracy. As one can see from our solution, the determined value of $M=m_{a}+m_{b}$ and correspondence between $l_{a}$ and $l_{b}$ in any quantum state means that both electrons of the shell are strongly correlated during their "motion" around nucleus. The ground state of the helium shell with $m_{a}=-m_{b}$ and $l_{a}=l_{b}$ means that two individual electrons are in equivalent quantum states, and $z$ projections of their angular momenta are oriented strongly oppositely. The wave function of the ground state is spatially symmetric, so that spins of the electrons in this state are in opposite orientations. This situation can be characterized exactly as pairing of the equivalent electrons within the unitary shell.

No low-energy excitations appear under external electric field. No nonlinear polarization effects up to atomically high fields can be predicted. Helium atom (as any other electrically neutral system) responds to external field by formation of intrinsic dipole moment which is a function of external electric field through field-dependent polarizability. However, a radical reconstruction of spectrum is inevitable in condensed helium phases (liquid and solid) because of an essential effect from fields of neighboring atoms. Thus, we can expect an appearance of low-energy excitations in helium condensed phases. Such excitations should be manifested through details of interatomic interactions in liquid and solid helium. To describe this effects theoretically we need to solve corresponding many-particle quantum-mechanical problem. This program will be a subject of further researches.

\section{Appendix 1}

After integration over radial variables we have

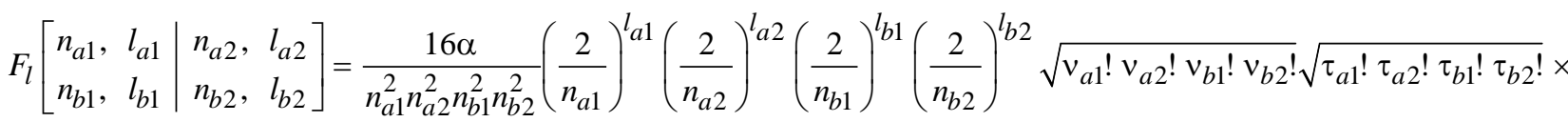

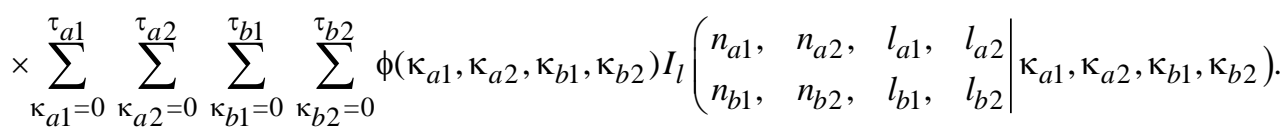

Here $\tau_{a}=n_{a}-l_{a}-1, \tau_{b}=n_{b}-l_{b}-1, v_{a}=n_{a}+l_{a}, v_{b}=n_{b}+l_{b}$, where $v_{a}=n_{a}+l_{a}, \quad v_{b}=n_{b}+l_{b}$,

$$
\begin{gathered}
\phi\left(\kappa_{a 1}, \kappa_{a 2}, \kappa_{b 1}, \kappa_{b 2}\right)=\left(\frac{2}{n_{a 1}}\right)^{\kappa_{a 1}}\left(\frac{2}{n_{a 2}}\right)^{\kappa_{a 2}}\left(\frac{2}{n_{b 1}}\right)^{\kappa_{b 1}}\left(\frac{2}{n_{b 2}}\right)^{\kappa_{b 2}} \frac{(-1)^{\kappa_{a 1}+\kappa_{a 2}+\kappa_{b 1}+\kappa_{b 2}}}{\kappa_{a 1} ! \kappa_{a 2} ! \kappa_{b 1} ! \kappa_{b 2} !} \times \\
\times \frac{1}{\left(\lambda_{a 1}+\kappa_{a 1}\right) !\left(\lambda_{a 2}+\kappa_{a 2}\right) !\left(\lambda_{b 1}+\kappa_{b 1}\right) !\left(\lambda_{b 2}+\kappa_{b 2}\right) !\left(\tau_{a 1}-\kappa_{a 1}\right) !\left(\tau_{a 2}-\kappa_{a 2}\right) !\left(\tau_{b 1}-\kappa_{b 1}\right) !\left(\tau_{b 2}-\kappa_{b 2}\right) !},
\end{gathered}
$$

with $\lambda_{a}=2 l_{a}+1, \quad \lambda_{b}=2 l_{b}+1$, and

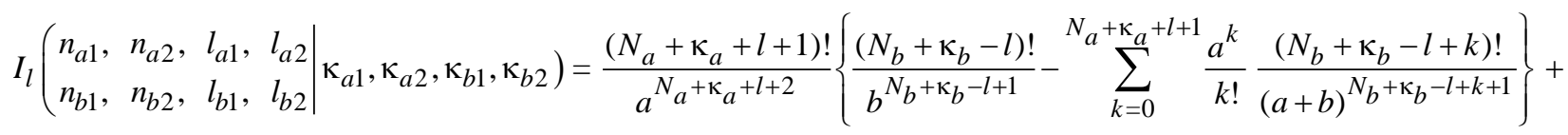


$+\frac{\left(N_{a}+\kappa_{a}-l\right) !}{a^{N_{a}+\kappa_{a}-l+1}} \sum_{k=0}^{N} \frac{a^{+\kappa_{a}-l}}{k !} \frac{\left(N_{b}+\kappa_{b}+l+k+1\right) !}{(a+b)^{N_{b}+\kappa_{b}+l+k+2}}=\frac{\left(N_{b}+\kappa_{b}+l+1\right) !}{b^{N_{b^{+}} \kappa_{b}+l+2}}\left\{\frac{\left(N_{a}+\kappa_{a}-l\right) !}{a^{N_{a}+\kappa_{a}-l+1}}-\sum_{k=0}^{N_{b}+\kappa_{b}+l+1} \frac{b^{k}}{k !} \frac{\left(N_{a}+\kappa_{a}-l+k\right) !}{(a+b)^{N_{a}+\kappa_{a}-l+k+1}}\right\}+$

$$
+\frac{\left(N_{b}+\kappa_{b}-l\right) !}{b^{N_{b}+\kappa_{b}-l+1}} \sum_{k=0}^{N_{b}+\kappa_{b}-l} \frac{b^{k}}{k !} \frac{\left(N_{a}+\kappa_{a}+l+k+1\right) !}{(a+b)^{N_{a}+\kappa_{a}+l+k+2}}
$$

where

$$
a=\frac{1}{n_{a 1}}+\frac{1}{n_{a 2}}, b=\frac{1}{n_{b 1}}+\frac{1}{n_{b 2}}, N_{a}=l_{a 1}+l_{a 2}+1, N_{b}=l_{b 1}+l_{b 2}+1, \kappa_{a}=\kappa_{a 1}+\kappa_{a 2}, \kappa_{b}=\kappa_{b 1}+\kappa_{b 2} .
$$

The result (42) is invariant relative to simultaneously replacement $a \Leftrightarrow b$ in all factors and indexes.

\section{Appendix 2}

We have

$$
\begin{gathered}
\mathcal{I}_{s}\left(n_{1}, l_{1} \mid n_{2}, l_{2}\right)=\int_{0}^{\infty} d r r^{2+s} R_{n_{1} l_{1}}(\alpha r) R_{n_{2} l_{2}}(\alpha r)=\frac{4}{\alpha^{s} n_{1}^{2} n_{2}^{2}} \sqrt{v_{1} ! v_{2} !} \sqrt{\tau_{1} ! \tau_{2} !}\left(\frac{2}{n_{1}}\right)^{l_{1}}\left(\frac{2}{n_{2}}\right)^{l_{2}} \times \\
\times \sum_{k_{1}=0}^{\tau_{1}} \sum_{k_{2}=0}^{\tau_{2}}\left(\frac{2}{n_{1}}\right)^{k_{1}}\left(\frac{2}{n_{2}}\right)^{k_{2}} \frac{(-1)^{k_{1}+k_{2}}}{k_{1} ! k_{2} !} \frac{\left(k_{1}+k_{2}+l_{1}+l_{2}+s+2\right) !}{a^{k_{1}+k_{2}+l_{1}+l_{2}+s+3}} \frac{1}{\left(\lambda_{a 1}+k_{1}\right) !\left(\lambda_{a 2}+k_{2}\right) !\left(\tau_{a 1}-k_{1}\right) !\left(\tau_{a 2}-k_{2}\right) !} .
\end{gathered}
$$

The integral is regular at $s>-2$.

\section{Appendix 3}

The functions of the basis set are

$$
\begin{aligned}
& u_{1}\left(\mathbf{r}_{a}, \mathbf{r}_{b}\right)=u_{100}\left(\alpha \mathbf{r}_{a}\right) u_{100}\left(\alpha \mathbf{r}_{b}\right) \equiv\left|\begin{array}{lll}
1 & 0 & 0 \\
1 & 0 & 0
\end{array}\right\rangle, \\
& u_{2}\left(\mathbf{r}_{a}, \mathbf{r}_{b}\right)=\left|\begin{array}{lll}
1 & 0 & 0 \\
2 & 0 & 0
\end{array}\right\rangle, \quad u_{3}\left(\mathbf{r}_{a}, \mathbf{r}_{b}\right)=\left|\begin{array}{lll}
2 & 0 & 0 \\
1 & 0 & 0
\end{array}\right\rangle \text {, } \\
& u_{4}\left(\mathbf{r}_{a}, \mathbf{r}_{b}\right)=\left|\begin{array}{lll}
1 & 0 & 0 \\
2 & 1 & 0
\end{array}\right|, \quad u_{5}\left(\mathbf{r}_{a}, \mathbf{r}_{b}\right)=\left|\begin{array}{lll}
2 & 1 & 0 \\
1 & 0 & 0
\end{array}\right\rangle \text {, } \\
& u_{6}\left(\mathbf{r}_{a}, \mathbf{r}_{b}\right)=\left|\begin{array}{ccc}
1 & 0 & 0 \\
2 & 1 & -1
\end{array}\right\rangle, \quad u_{7}\left(\mathbf{r}_{a}, \mathbf{r}_{b}\right)=\left|\begin{array}{ccc}
2 & 1 & -1 \\
1 & 0 & 0
\end{array}\right\rangle \text {, } \\
& u_{8}\left(\mathbf{r}_{a}, \mathbf{r}_{b}\right)=\left|\begin{array}{lll}
1 & 0 & 0 \\
2 & 1 & 1
\end{array}\right|, \quad u_{9}\left(\mathbf{r}_{a}, \mathbf{r}_{b}\right)=\left|\begin{array}{lll}
2 & 1 & 1 \\
1 & 0 & 0
\end{array}\right| \text {, } \\
& u_{10}\left(\mathbf{r}_{a}, \mathbf{r}_{b}\right)=\left|\begin{array}{lll}
2 & 0 & 0 \\
2 & 0 & 0
\end{array}\right\rangle, \quad u_{11}\left(\mathbf{r}_{a}, \mathbf{r}_{b}\right)=\left|\begin{array}{lll}
2 & 0 & 0 \\
2 & 1 & 0
\end{array}\right\rangle \text {, } \\
& u_{12}\left(\mathbf{r}_{a}, \mathbf{r}_{b}\right)=\left|\begin{array}{lll}
2 & 1 & 0 \\
2 & 0 & 0
\end{array}\right\rangle, \quad u_{13}\left(\mathbf{r}_{a}, \mathbf{r}_{b}\right)=\left|\begin{array}{ccc}
2 & 0 & 0 \\
2 & 1 & -1
\end{array}\right\rangle \text {, } \\
& u_{14}\left(\mathbf{r}_{a}, \mathbf{r}_{b}\right)=\left|\begin{array}{ccc}
2 & 1 & -1 \\
2 & 0 & 0
\end{array}\right\rangle, \quad u_{15}\left(\mathbf{r}_{a}, \mathbf{r}_{b}\right)=\left|\begin{array}{lll}
2 & 0 & 0 \\
2 & 1 & 1
\end{array}\right\rangle \text {, } \\
& u_{16}\left(\mathbf{r}_{a}, \mathbf{r}_{b}\right)=\left|\begin{array}{lll}
2 & 1 & 1 \\
1 & 0 & 0
\end{array}\right\rangle, \quad u_{17}\left(\mathbf{r}_{a}, \mathbf{r}_{b}\right)=\left|\begin{array}{lll}
2 & 1 & 0 \\
2 & 1 & 0
\end{array}\right\rangle \text {, }
\end{aligned}
$$$$
u_{18}\left(\mathbf{r}_{a}, \mathbf{r}_{b}\right)=\left|\begin{array}{ccc}
2 & 1 & 0 \\
2 & 1 & -1
\end{array}\right\rangle, \quad u_{19}\left(\mathbf{r}_{a}, \mathbf{r}_{b}\right)=\left|\begin{array}{ccc}
2 & 1 & -1 \\
2 & 1 & 0
\end{array}\right|,
$$$$
u_{20}\left(\mathbf{r}_{a}, \mathbf{r}_{b}\right)=\left|\begin{array}{lll}
2 & 1 & 0 \\
2 & 1 & 1
\end{array}\right|, \quad u_{21}\left(\mathbf{r}_{a}, \mathbf{r}_{b}\right)=\left|\begin{array}{lll}
2 & 1 & 1 \\
2 & 1 & 0
\end{array}\right\rangle \text {, }
$$$$
u_{22}\left(\mathbf{r}_{a}, \mathbf{r}_{b}\right)=\left|\begin{array}{ccc}
2 & 1 & -1 \\
2 & 1 & -1
\end{array}\right\rangle, \quad u_{23}\left(\mathbf{r}_{a}, \mathbf{r}_{b}\right)=\left|\begin{array}{ccc}
2 & 1 & -1 \\
2 & 1 & 1
\end{array}\right\rangle \text {, }
$$$$
u_{24}\left(\mathbf{r}_{a}, \mathbf{r}_{b}\right)=\left|\begin{array}{ccc}
2 & 1 & 1 \\
2 & 1 & -1
\end{array}\right|, \quad u_{25}\left(\mathbf{r}_{a}, \mathbf{r}_{b}\right)=\left|\begin{array}{lll}
2 & 1 & 1 \\
2 & 1 & 1
\end{array}\right| \text {. }
$$

\section{Appendix 4}

The rest of eigenfunctions for solution with $N=25$ is

$$
\begin{aligned}
& \Psi_{(4)}\left(\mathbf{r}_{a}, \mathbf{r}_{b}\right)=0.7066\left[u_{4}\left(\mathbf{r}_{a}, \mathbf{r}_{b}\right)-u_{5}\left(\mathbf{r}_{a}, \mathbf{r}_{b}\right)\right]+ \\
& +0.02696\left[u_{11}\left(\mathbf{r}_{a}, \mathbf{r}_{b}\right)-u_{12}\left(\mathbf{r}_{a}, \mathbf{r}_{b}\right)\right] \text {, } \\
& \Psi_{(5)}\left(\mathbf{r}_{a}, \mathbf{r}_{b}\right)=0.70538\left[u_{4}\left(\mathbf{r}_{a}, \mathbf{r}_{b}\right)+u_{5}\left(\mathbf{r}_{a}, \mathbf{r}_{b}\right)\right]+ \\
& +0.0494\left[u_{11}\left(\mathbf{r}_{a}, \mathbf{r}_{b}\right)+u_{12}\left(\mathbf{r}_{a}, \mathbf{r}_{b}\right)\right], \\
& \Psi_{(6)}\left(\mathbf{r}_{a}, \mathbf{r}_{b}\right)=0.70655\left[u_{6}\left(\mathbf{r}_{a}, \mathbf{r}_{b}\right)-u_{7}\left(\mathbf{r}_{a}, \mathbf{r}_{b}\right)\right]+ \\
& +0.02806\left[u_{13}\left(\mathbf{r}_{a}, \mathbf{r}_{b}\right)-u_{14}\left(\mathbf{r}_{a}, \mathbf{r}_{b}\right)\right] \text {, }
\end{aligned}
$$




$$
\begin{gathered}
\Psi_{(7)}\left(\mathbf{r}_{a}, \mathbf{r}_{b}\right)=0.7055\left[u_{6}\left(\mathbf{r}_{a}, \mathbf{r}_{b}\right)+u_{7}\left(\mathbf{r}_{a}, \mathbf{r}_{b}\right)\right]+ \\
+0.04762\left[u_{13}\left(\mathbf{r}_{a}, \mathbf{r}_{b}\right)+u_{14}\left(\mathbf{r}_{a}, \mathbf{r}_{b}\right)\right], \\
\Psi_{(8)}\left(\mathbf{r}_{a}, \mathbf{r}_{b}\right)=0.7065\left[u_{8}\left(\mathbf{r}_{a}, \mathbf{r}_{b}\right)-u_{9}\left(\mathbf{r}_{a}, \mathbf{r}_{b}\right)\right]+ \\
+0.0281\left[u_{15}\left(\mathbf{r}_{a}, \mathbf{r}_{b}\right)-u_{16}\left(\mathbf{r}_{a}, \mathbf{r}_{b}\right)\right], \\
\Psi_{(9)}\left(\mathbf{r}_{a}, \mathbf{r}_{b}\right)=0.7055\left[u_{8}\left(\mathbf{r}_{a}, \mathbf{r}_{b}\right)+u_{9}\left(\mathbf{r}_{a}, \mathbf{r}_{b}\right)\right]+ \\
+0.04762\left[u_{15}\left(\mathbf{r}_{a}, \mathbf{r}_{b}\right)+u_{16}\left(\mathbf{r}_{a}, \mathbf{r}_{b}\right)\right] .
\end{gathered}
$$

1. A.S. Rybalko, Fiz. Nizk. Temp. 30, 1321 (2004) [Low Temp. Phys. 30, 994 (2004)].

2. A.S. Rybalko and S.P. Rubets, Fiz. Nizk. Temp. 31, 820 (2005) [Low Temp. Phys. 31, 623 (2005)].

3. A. Rybalko, S. Rubets, E. Rudavskii, V. Tikhy, S. Tarapov, R. Golovashchenko, and V. Derkach, Phys. Rev. B 76, 140503 (2007).

4. A. Rybalko, S. Rubets, E. Rudavskii, V. Tikhy, V. Derkach, and S. Tarapov, J. Low Temp. Phys. 148, 527 (2007).

5. A.S. Rybalko, S.P. Rubets, E.Ya. Rudavskii, V.A. Tikhy, S.I. Tarapov, R.V. Golovashchenko, and V.N. Derkach, Fiz. Nizk. Temp. 34, 326 (2008) [Low Temp. Phys. 34, 254 (2008)].

6. A.S. Rybalko, S.P. Rubets, E.Ya. Rudavskii, V.A. Tikhy, S.I. Tarapov, R.V. Golovashchenko, and V.N. Derkach, Fiz. Nizk. Temp. 34, 631 (2008) [Low Temp. Phys. 34, 497 (2008)].

7. A. Rybalko, S. Rubets, E. Rudavskii, V. Tikhiy, Y. Poluectov, R. Golovashchenko, V. Derkach, S. Tarapov, and O. Usatenko, Fiz. Nizk. Temp. 35, 1073 (2009) [Low Temp. Phys. 35, 837 (2009)].

8. A. Rybalko, S. Rubets, E. Rudavskii, V. Tikhiy, Y. Poluectov, R. Golovashchenko, V. Derkach, S. Tarapov, and O. Usatenko, J. Low Temp. Phys. 158, 244 (2010).

9. H.A. Bethe and E.E. Salpeter, Quantum Mechanics of Oneand Two-Electron Atoms, Plenum, NY (1977).

10. P. Gombaś, Theorie und Lösungsmethoden des Mehrteilchenproblems der Wellenmechanik, Basel (1950).

11. G. Tanner, K. Richter, and J.-M. Rost, Rev. Mod. Phys. 72, 497 (2000).

12. G.W.F. Drake, in: The Hydrogen Atom, S.G. Karshenboim et al. (eds.), Springer-Verlag Berlin-Heidelberg (2001), p. 57.

13. E.A. Hylleraas, Z. Phys. 54, 347 (1929).

14. T. Kinoshita, Phys. Rev. 105, 1490 (1957).

15. T. Kinoshita, Phys. Rev. 115, 366 (1959).

16. G.W.F. Drake, Nucl. Inst. Meth. Phys. Res. B 31, 7 (1998).

17. P. Walsh and S. Borowitz, Phys. Rev. 115, 1206 (1959).

18. C.L. Pekeris, Phys. Rev. 112, 1649 (1958).

19. C.L. Pekeris, Phys. Rev. 115, 1216 (1959).

20. E.R. Davidson, J. Cem. Phys. 39, 875 (1963).

21. G.R. Taylor and R.G. Parr, Proc. Natl. Acad. Sci. U.S. 38, 154 (1952).
22. A.M. Frolov, Sov. Phys. JETP 65, 1100 (1987).

23. E.A. Hylleraas, Z. Phys. 65, 209 (1930).

24. R.E. Knight, C.W. Scherr, Phys. Rev. 128, 2675 (1962).

25. C.W. Scherr and R.E. Knight, Rev. Mod. Phys. 35, 436 (1963).

26. J.G. Leopold, I.C. Percival, and A.S. Tworkowski, J. Phys. B: At. Mol. Phys. 13, 1025 (1980).

27. J.G. Leopold and I.C. Percival, J. Phys. B: At. Mol. Phys. 13, 1037 (1980).

28. D.R. Hartree, Proc. Cambr. Phil. Soc. 26, 89 (1928).

29. V. Fock, Z. Phys. 61, 126 (1930).

30. V. Fock, Z. Phys. 62, 795 (1930).

31. V. Fock and M.J. Petrashen, Phys. Zs. Sowjetunion 6, 368 (1934).

32. F. Seitz, Modern Theory of Solids, McGraw-Hill Co., NY (1940).

33. H. Mitler, Phys. Rev. 99, 1835 (1955).

34. D.R. Hartree, The Calculation of Atomic Structures, John Wiley, New York (1957).

35. R. Courant and D. Hilbert, Methods of Mathematical Physics, Interscience Publishers, NY (1953).

36. P.M. Morse and H. Feshbach, Methods of Theoretical Physics, McGraw-Hill, NY (1953).

37. S. Flügge, Practical Quantum Mechanics II, Springer (1971).

38. W.R. Johnson, Lectures on Atomic Physics, Univ. of Notre Dame, Indiana, USA (2006).

39. A.D. Buckingham and P.G. Hibbard, Symp. Faraday Soc. 2, 41 (1968).

40. J.E. Rice, P.R. Taylor, T.J. Lee, and J. Alm, J. Chem Phys. 94, 4972 (1991).

41. B.N. Parlett, The Symmetric Eigenvalue Problem, PrenticeHall, Inc., NY (1980).

42. J.H. Wilkinson, Algebraic Eigenvalue Problem, Oxford Univ. Press, London (1965).

43. J.K.L. MacDonald, Phys. Rev. 43, 830 (1933).

44. S.H. Gould, Variational Methods for Eigenvalue Problems, Oxford Univ. Press, London (1966).

45. J. Frenkel, Einführung in die Wellenmechanik, Julius Springer, Berlin (1929).

46. L.D. Landau and E.M. Lifshitz, Quantum Mechanic: Nonrelativistic Theory, Pergamon Press, Oxford (1965).

47. D.A. Varshalovich, A.N. Moskalev, and V.K. Khersonski, Quantum Theory of Angular Momentum, World Scientific, Singapore (1988).

48. A. Messiah, Quantum Mechanics II, North Holland, Amsterdam (1961).

49. J.H. Wilkinson and R. Reinsch, Handbook for Automatic Computation: Linear Algebra, Springer, Berlin (1971).

50. A.A. Radzig and B.M. Smirnov, Handbook for Atomic and Molecular Physics, Atomizdat, Moscow (1980) [in Russian]. 